\title{
A Trans-equatorial Filament and the Bastille Day Flare/CME Event
}

\author{
Jingxiu Wang, Guiping Zhou, Yayuan Wen, Yuzong Zhang, \\ Jun Zhang, Huaning Wang, and Yuanyong Deng
}

National Astronomical Observatories, Chinese Academy of Sciences, Beijing 100012, China

(email: wjx@ourstar.bao.ac.cn)

\begin{abstract}
The Bastille Day Event on July 14 2000, a major solar flare and a global coronal mass ejection (CME), is not a phenomenon of a single active region. Activation and eruption of a huge trans-equatorial filament is seen to precede the simultaneous filament eruption and flare in the source active region, AR9077, and the full halo-CME in the high corona. Evidence of reconfiguration of large-scale structures, manifested by SOHO EIT and Yohkoh SXT observations, is clearly seen. The large-scale magnetic composition related to the trans-equatorial filament and its sheared magnetic arcades appears to be the essential part of the CME parent magnetic structure. Estimations show that the filament-arcade system has enough magnetic helicity to account for the helicity carried by the related CMEs.
\end{abstract}

Keywords. Sun: coronal mass ejections (CMEs), Sun: filaments, Sun: activity

\section{Activity of the Transequatorial Filament}

To understand CME magnetism, the observations of Bastille Day event (Zhang et al. 2001; Deng et al. 2001) have been re-examined. It is found that one fact has been missing in the published literature: the pre-CME activation and eruption of a huge transequatorial filament. The active region filament in AR9077 could be considered as the extension, or rooting end of this huge filament.

A huge trans-equatorial filament was mapped by the Huairou full disk $\mathrm{H} \alpha$ filtergraph equipped with a $2 \mathrm{k} \times 2 \mathrm{k}$ CCD. Figure 1 shows the filament in comparison with its background magnetic field from MDI magnetogram and coronal structures from SOHO EIT and Yohkoh SXT images. The huge filament divided an extended bipolar region (EBR) which included the magnetic flux of AR9077, 9082 and several smaller ARs, as well as enhanced network.

The filament often twisted, knotted, kinked, or partial disappeared. Disturbances started from the filament in AR9082 on the southern hemisphere, was responded by the huge filament and propagated into AR9077. The global eruption of the trans-equatorial filament started approximately from 08:22 UT and earliest brightening in the huge filament was seen at 09:02 UT when the filament tied-up closely to its northern end. The filament in AR9077 began to break out and erupt since 10:02 UT, in coincidence with the beginning of Bastille Day flare.

\section{Reconfiguration of Large-scale Magnetic Structure}

The reconfiguration of large-scale magnetic structure appeared as followings. First, the arcades straddling over the trans-equatorial filament disappeared; Secondly, there appeared dimming at both EUV and soft X-ray wavelengths at 3 different locations 


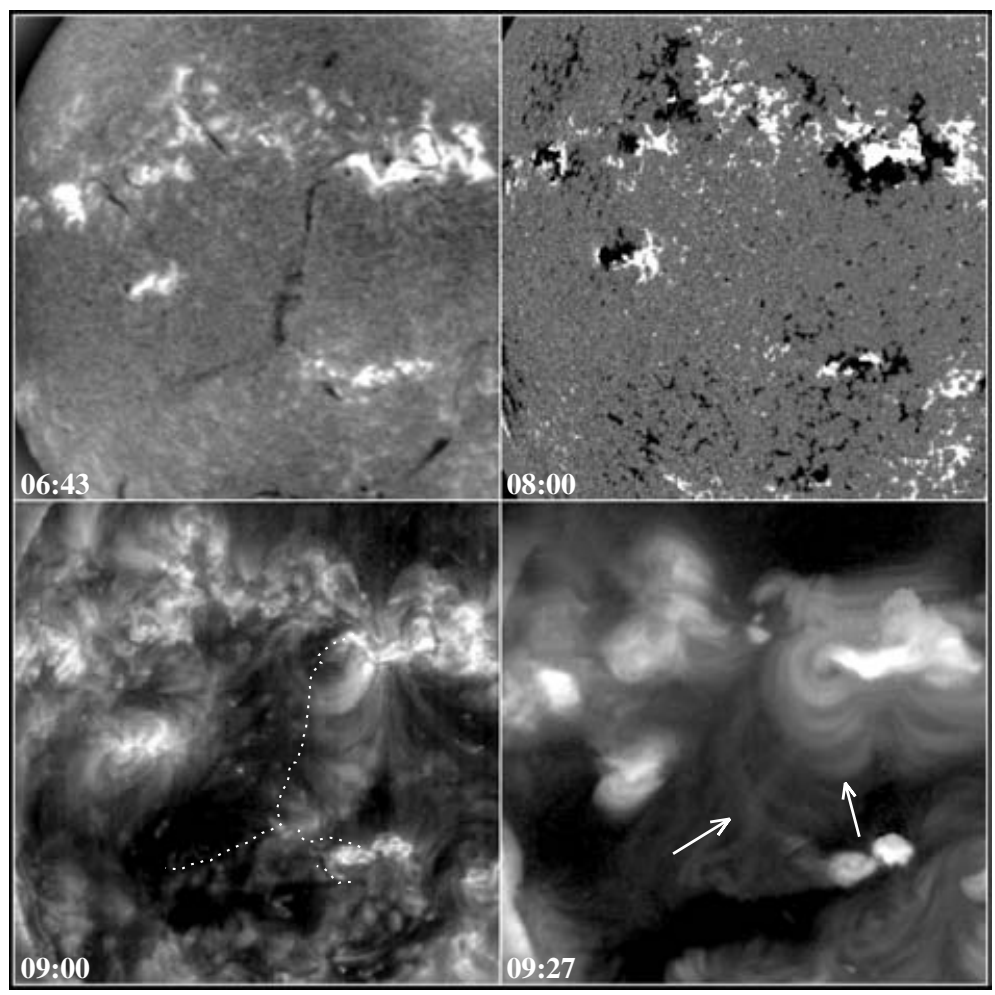

Figure 1. Upper-left: $\mathrm{H} \alpha$ filtergram showing the huge trans-equatorial filament; Upper-right: MDI magnetogram; Lower-left: EIT image showing the arcade system striding over the filament; Lower-right: SXT image showing the arcade system indicated by two arrows.

which were widely separated. It was not transient in nature, but manifested some longterm restructuring of the Sun's magnetic fields after the flare/CME event. The widely separated dimming seems to imply that not only the AR9077, but also the multiple flux systems covering a broad spatial range are involved in the physical process which led to the major activity.

Non-linear force-free extrapolations based on the MDI magnetograms show the magnetic arcades overlapping the trans-equatorial filament changed from reversed 'S'-shaped stressed structure before the flare/CME to more relaxed state. A gross estimation finds that the helicity of sheared arcades is approximate $3 \times 10^{43} M x^{2}$. It seems to be enough to account for the helicity source of the CME.

\section{Acknowledgements}

The work is supported by the National Natural Science Foundation of China(10233050) and the National Key Basic Science Foundation(TG2000078404).

\section{References}

Deng, Y., Wang, J., Yan, Y., \& Zhang, J. 2001, Solar Phys 204, 11-26.

Zhang, J., Wang, J., Deng, Y., \& Wu, D. 2001, ApJ 548, L99-102. 